\title{
Theoretical Models for Predicting the Effect of Bridging Group Recognition and Conjugate Substitution on Hapten Enzyme Immunoassay Dose-Response Curves ${ }^{1}$
}

\author{
LEONidas G. BaChas ${ }^{2}$ ANd Mark E. MEYERHOFF \\ Department of Chemistry, University of Michigan, Ann Arbor, Michigan 48109
}

Received September 3, 1985

\begin{abstract}
Models for predicting the effect of immunological recognition of the bridge group on the doseresponse curves obtained with heterogeneous hapten enzyme immunoassays are presented. Appropriate theoretical treatment shows that the greater affinity of antibodies toward the enzymclabeled species than for the unlabeled hapten analyte results in assays with limited detection capabilities. This problem is compounded when enzyme conjugates possessing multiple haptens are used. In equilibrium type competitive arrangements, the concentrations of binder and labeled hapten may be optimized to some extent to improve assay performance. However. the results presented show that only when assays are performed in a sequential binding mode using carefully controlled timing of reagent incubations can the detection capabilities of the assays be fully maximized for analyte measurements. Unfortunately, it is also shown that such sequential binding approaches render the assays essentially nonselective. The effect of decreasing the affinity of the binder to the enzyme-labeled hapten relative to the unlabeled analyte by using heterologous conjugates in equilibrium arrangements is shown to improve detection capabilities but also at the expense of reduced selectivity. Suggestions for reagent concentrations and conjugate substitution (degree of conjugation), which provide optimized dose-response curves at a given $\mathrm{ED}_{50}$ value, are also presented as are proposals for using different binders which do not exhibit bridging group recognition. 1986 Academic Press. Inc.

KEY WORDS: binding assays: immunochemical methods: enzyme labels; bridging group recognition; enzyme conjugate substitution.
\end{abstract}

The use of enzymes rather than radioisotopes as labels in competitive binding assays has resulted in the development of a wide range of new enzyme immunoassay (EIA) ${ }^{3}$ techniques for measurement of important biological molecules (1-3). While the use of enzymes may overcome some of the costs and hazards associated with conventional radioimmunoassay (RIA) procedures, the detection capabilities of such methods can often be limited. In some cases the detection limits can be drastically affected by antibody recognition of the bridging group which cova-

\footnotetext{
' This work was supported under Grants CHE-818817 and CHE-8506695 from the National Science Foundation.

${ }^{2}$ Present Address: Dept. of Chemistry, University of Kentucky, Lexington, KY 40506.

${ }^{3}$ Abbreviations used: EIA, enzyme immunoassay; RIA, radioimmunoassay.
}

lently links the hapten (ligand) to the enzyme label (4-9). Since this same link is usually utilized in the preparation of hapten protein immunogens used to elicit antibody formation, the resulting antibodies typically have a much higher affinity toward the enzyme-labeled hapten than for unlabeled analyte. The purpose of this report is to theoretically (based on a mass-action model) predict the behavior of assays in which this dissimilarity in binding affinities exists and to examine how variation in reagent concentrations and actual methodology (equilibrium vs sequential binding) can affect the dose-response curves. An approach for optimizing reagent concentrations in equilibrium methods is also presented.

Bridging group recognition problems are also often observed in classical RIA procedures, particularly when there is no method 
for directly radiolabeling the haptenic species (10-12). For example, in RIAs of steroids, hemisuccinate derivatives of the steroids are used to couple these molecules to radioiodinated tyrosine to form the labeled reagent. The same hemisuccinate derivatives are also used to prepare the immunogen needed for antibody production, and in these reports it is clearly shown that the resulting antisera have a higher affinity for the hemisuccinate derivative than for the unmodified steroid. Similar problems can occur in newer fluoroimmunoassay methods where the fluorescent tag must be covalently linked to hapten to form the labeled reagent ( 7 ). However, the problem is magnified considerably in EIAs since the derivatized hapten is typically linked to the enzyme through a lysine residue. Thus, aside from the derivatized arm of the hapten (e.g., hemisuccinate) the bridging group also includes this lysine residue of the protein. Since the original immunogen also possesses the same derivative-lysine link, antibodies are likely to have an even greater affinity for labels containing the exact same sequence of bonds (i.e., enzyme-hapten conjugates). In general, the smaller the hapten species, the greater the chances of significant bridging group recognition problems.

Although theoretical treatments have already been reported for the case where labeled and unlabeled ligands demonstrate different affinities for the binder in immunoassays (1316), these results apply to techniques which employ monosubstituted labeled species, such as RIA and fluoroimmunoassay. However, it is very common that the enzyme conjugates used in hapten EIAs are multivalent, with more than one ligand attached per enzyme molecule. The number of hapten molecules per molecule of enzyme depends on the conditions of the coupling reaction, as well as on the nature of both the enzyme and the hapten, and may vary from $1: 1$ to $30: 1$ or more. In this case the effective concentration of the labeled ligand (i.e., ligand molecules attached to sites on the enzyme that are stcrcochcmically unrestricted to approach by the binder) is in- creased. Thus, the statistical association constant between the binder and the labeled species is increased, which further enhances the effect of bridging group recognition on assay performance.

Aside from the multiplicity issue, our theoretical analysis is based on plotting dose-response curves as bound vs logarithm of analyte concentration. While this type of plotting of EIA results is very common, there is a lack of theoretical treatment of this case, particularly when associated with the effect of bridging group recognition and multiple substitution of the label on assay performance. Furthermore, in this report there is a theoretical approach to nonequilibrium curves obtained when sequential binding methodologies are employed. Such a theoretical treatment is unique in the case of unequal binding affinities and, in view of the suggested advantages of the sequential binding approach for overcoming bridging group recognition, may aid in optimizing this alternate method.

Researchers have already proposed practical approaches to solve the bridging recognition problem in EIAs $(4-6,17,18)$. The most logical approach is to reduce the affinity (association) constant between the labeled species and the antibody binder. Typically this is done by using a structurally different bridge for preparing the labeled species than was used in the original immunogen $(5,6)$. Empirically, it has been shown that this heterologous approach can improve the assay response toward the analyte but often decrease the selectivity of the assay. We now demonstrate that this decrease in selectivity can be predicted based on appropriate theoretical treatment of the relative change in affinity between the antibody and the labeled species, the analyte, and the interferent (e.g., cross-reacting substance).

\section{NOMENCLATURE}

$p$, total concentration of unlabeled ligand in reaction volume

$p^{*}, \quad$ total concentration of labeled ligand in reaction volume 
q, total concentration of binding sites in reaction volume

$K, \quad$ equilibrium affinity (association) constant of binder-ligand reaction $\left(K=k_{1} / k_{-1}\right)$

$K^{*}, \quad$ equilibrium affinity constant of association reaction of binder with labeled ligand $\left(K^{*}=k_{2} / k_{-2}\right)$

$k_{i}, \quad$ association rate constant $(i=1,2)$

$k_{-i}, \quad$ dissociation rate constant $(i=1,2)$

[QP], concentration of binder-ligand complex

[QP*]. concentration of complex of labeled

ligand with the binder

[P]. concentration of free ligand

$\left[\mathrm{P}^{*}\right], \quad$ concentration of free labeled ligand

[Q], concentration of unoccupied bind-

ing sites

$\mathrm{EP}_{n}, \quad$ multivalent conjugate

$n, \quad$ effective conjugation ratio

$\mathrm{QEP}_{n}, \quad$ complex of multivalent conjugate

with binder

$K_{\text {sla1 }}$, equilibrium affinity constant of association reaction of binder with multivalent conjugate

$t$, duration of second incubation of sequential binding assays

$b_{0}, \quad$ response for the zero standard tube

$K b_{0}$, response for the zero standard tube normalized with respect to $K$

$\mathrm{ED}_{50}$, effective dose for $50 \%$ of the maximum response

$\mathrm{KED}_{50}$, effective dose for $50 \%$ of the maximum response normalized with respect to $K$

ID 50 , dose of inhibitor for $50 \%$ inhibition of the maximum response

$K_{\mathrm{I}}$, association constant of binder-interferent reaction

\section{MODELS FOR HETEROGENEOUS ENZYME-LINKED ASSAYS}

Equilibrium mode. The principle of singleincubation enzyme-linked assays (otherwise called equilibrium mode or simultaneous addition assays) is based on the competition of the enzyme-hapten conjugate and the unla- beled hapten (sample or standard) for a fixed number of binding sites of the specific binder (19). The higher the unlabeled hapten concentration in the sample, the less conjugate is bound to the binder. Another protocol involves a preincubation of the specific binder with unlabeled ligand (analyte) followed by addition of the enzyme conjugate in a sequential binding technique (otherwise referred to as delayed addition, or nonequilibrium, or two-step assay) (20). In this case, the amount of bound enzyme conjugate is also inversely related to the quantity of analyte. The protocols for both of these assays usually involve some type of separation step after an appropriate incubation time (heterogeneous assays). This is accomplished either by immobilizing the antibody to a solid support or by using immobilized second antibody which has high affinity for the hapten-specific antibody (21). Once the activity of the enzyme is determined in the solid phase, a dose-response curve is plotted from the data obtained with standards, and the concentration of the unknown is inferred from this curve.

For the single-incubation enzyme immunoassay technique, labeled and unlabeled haptens are mixed first, followed by addition of the antibody and subsequent incubation of the mixture until equilibrium is achieved. The equilibrium expressions for this approach can be formulated as

$$
\begin{array}{cc}
\mathrm{P}+\mathrm{Q} & \rightleftharpoons \mathrm{QP}, \quad K \\
\mathrm{P}^{*}+\mathrm{Q} & \rightleftharpoons \mathrm{QP}^{*}, \quad K^{*}
\end{array}
$$

where $P$ is the unlabeled hapten (standard or sample), $\mathrm{P}^{*}$ is the enzyme-hapten conjugate, and $\mathrm{Q}$ is the antibody. $K$ and $K^{*}$ are the association constants for the two reactions. The initial concentrations of $P, P^{*}$, and $Q$ will be symbolized by $p, p^{*}$, and $q$, respectively. The antibody will be considered immobilized on a solid support such that no dissociation of the antibody from the support can take place. In this way the separation of the bound and free fractions is accomplished in a single step. This assumption will be made throughout our 
theoretical treatment and will simplify the modeling efforts (do not need to consider second equilibrium binding process (22)).

For the case of single-step assays, dose-response curves were simulated assuming a single-incubation equilibrium system which employs a binder possessing univalent, independent, and equivalent binding sites (described in Appendix I). For EIAs, such assumptions are met if a divalent antibody can simultaneously bind two different enzyme-ligand conjugates. It was also assumed that the enzymc conjugate was purified so that it did not contain any unconjugated hapten, any unconjugated enzyme, or any conjugated inactive enzyme.

Equilibrium mode: Effect of conjugate substitution. To demonstrate the effect of the multivalency of the conjugate in the assay, the following model was used. The conjugate, $\mathrm{EP}_{n}$, was assumed to have an effective conjugation ratio (number of ligand molecules attached per enzyme molecule that can be bound by the binder) of $n$. Further, the binder can associate with any of the $n$ ligands that are conjugated to the same enzyme, but once the binder binds one of these ligands the other ligands become stereochemically restricted to such a binding and do not participate in any other equilibria. The last assumption is more likely true only when the binder is immobilized on solid support. Finally, the multivalent conjugate will be considered as having independent ligand molecules, each one having the same microscopic association constant (for definition, see Ref. (23)), $K^{*}$, for the binder. These assumptions result in the reaction scheme

$$
\begin{aligned}
\mathrm{P}+\mathrm{Q} & \rightleftharpoons \mathrm{QP}, \quad K \\
\mathrm{EP}_{n}+\mathrm{Q} & \rightleftharpoons \mathrm{QEP}_{n}, \quad K_{\text {stat }}=n K^{*}
\end{aligned}
$$

where Q, $\mathrm{P}, \mathrm{QP}, K$, and $K^{*}$ are as defined for Scheme [I], and $K_{\text {stat }}$ is the association constant of this multivalent conjugate with the binder. Because the binder may bind to any of the $n$ available ligands on the conjugate, it is statistically $n$ times as probable for the binder to associate with the conjugate, $K_{\text {stat }}=n K^{*}$. This value is an upper limit for $K_{\text {stat }}$. Due to steric hindrance or site-site interactions, $K_{\text {stat }}$ is likely to be much smaller.

Sequential binding mode. Sequential binding assays have been customarily utilized to develop assays with steeper dose-response curves when compared to equilibrium techniques (20). Mathematical analysis of the kinetics of this system has demonstrated the potential increase in the steepness of the curves inherent in this approach (24). Rodbard et al. (24) treated the case where the kinetics of the binding reactions for the labeled and unlabeled ligand are identical. However, this is not normally the case when there is bridging group recognition involved and theoretical treatment of this situation has been mentioned (24) but never fully evaluated.

The sequential binding assay involves a preincubation step of the unlabeled ligand with the binder,

$$
\mathrm{P}+\mathrm{Q} \underset{k_{-1}}{\stackrel{k_{1}}{\rightleftharpoons}} \mathrm{QP}, \quad K=k_{1} / k_{-1},
$$

followed by a second incubation after the labeled ligand has been added to the reaction mixture. During the second incubation both the equilibrium equations

$$
\begin{array}{cl}
\mathrm{P} & +\mathrm{Q} \underset{k-1}{\stackrel{k_{1}}{\rightleftharpoons} \mathrm{QP},} \quad K=k_{1} / k_{-1} \\
\mathrm{P}^{*}+\mathrm{Q} \underset{k_{-2}}{\stackrel{k_{2}}{\rightleftharpoons}} \mathrm{QP}^{*}, & K^{*}=k_{2} / k_{-2}
\end{array}
$$

hold; the various parameters of this system are as defined before for the equilibrium system, $k_{1}$ and $k_{2}$ are the association rate constants, and $k_{-1}$ and $k_{-2}$ are the dissociation rate constants of the two reactions.

The theoretical simulation of this principle is based on a kinetic model similar to that suggested by Rodbard et al. (24) where the first incubation is long enough to allow equilibrium to be reached, while during the second incubation

$$
\begin{gathered}
\frac{d[\mathrm{QP}]}{d t}=-k_{-1}[\mathrm{QP}]+k_{1}[\mathrm{P}][\mathrm{Q}] \\
\frac{d\left[\mathrm{QP}^{*}\right]}{d t}=-k_{-2}\left[\mathrm{QP}^{*}\right]+k_{2}\left[\mathrm{P}^{*}\right][\mathrm{Q}]
\end{gathered}
$$


The model calculates the parameters $[\mathrm{P}],[\mathrm{QP}]$, and [Q] for the first incubation which serve as initial values for the set of Eqs. [1] and [2] (see Appendix II).

For both assay modes (equilibrium and scquential binding), in order to have a more general treatment, unitless parameters were used for the simulations. This was accomplished by multiplying the various parameters with the association equilibrium constant, $K$, of the binder for the unlabeled analyte (e.g., dose-response curves of $K_{\text {bound }}$ vs $\log K p$ were generated instead of bound vs $\log p$ curves) or dividing by the amount of labeled ligand, $p^{*}$ (e.g., dose-response curves of bound $/ p^{*}$ vs log $K p$ were used) as described in the appendixes. These approaches allow a more direct comparison of assay performances under various conditions.

All data were obtained using programs written in Commodore BASIC and run on a Commodore CBM Model 8032 desk top computer.

\section{RESULTS AND DISCUSSION}

Several parameters have been used to define the "sensitivity" of immunoassay systems and this often results in misunderstandings within the competitive binding assay literature (2527) and in different conditions for optimized assays (19). To avoid such a confusion, the term sensitivity will not be used in the following discussion. Instead, to evaluate and compare the performance of each assay modeled. three different parameters will be used as indicated in Fig. 1: (a) the maximum response, $K b_{0}$ (i.e., the response at zero dose normalized with respect to $K$ ), (b) the $\mathrm{KED}_{50}$ value (i.e., the effective dose that produces $50 \%$ of the maximum response, $\mathrm{ED}_{50}$, again normalized with respect to $K$ ), and (c) the steepness of the dose-response curve. The steepness of the dose-response curve is defined as the maximum value of its slope (maximum value of $\left.d\left(K\left[\mathrm{QP}^{*}\right]\right) / d(\log K p)\right)$. This occurs at the inflection point of the dose-response curve. The relative term $b_{0} / p^{*}$ and the relative steepness

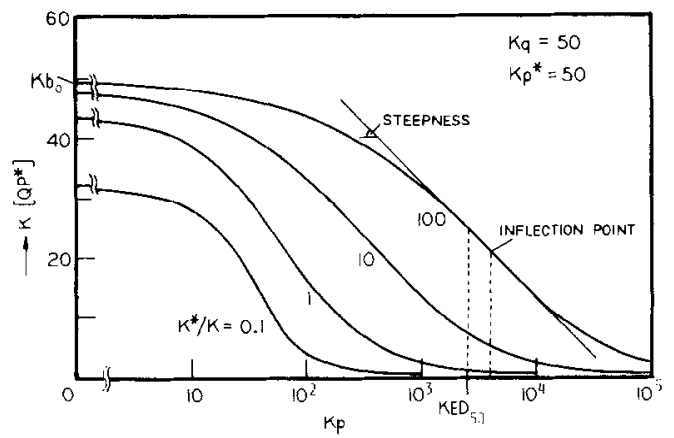

FIG. 1. Family of equilibrium dose-response curves for $K_{\text {bound }}\left(K\left[\mathrm{QP}^{*}\right]\right)$ as a function of the logarithm of normalized ligand concentration $(K p)$. The curves were generated with $K^{*} / K$ ratios of $0.1,1,10$, and 100 . Both parameters, $K q$ and $K p^{*}$, were fixed at 50 for all curves. The definitions of the various response parameters $\left(K b_{0}\right.$. $\mathrm{KED}_{50}$, and steepness) are also shown.

(maximum value of $d\left(\left[\mathrm{QP}^{*}\right] / p^{*}\right) / d(\log K p)$ ) are also used to evaluate the response characteristics of the assays. The $K b_{0}$ and $K E D_{50}$ as well as the steepness and relative steepness are calculated as shown in Appendix III. The $\mathrm{ED}_{50}$ value does not necessarily correspond to the inflection point of the dose-response curve. Nevertheless, it is customary to use $\mathrm{ED}_{50}$ values rather than inflection point values to evaluate dose-response curves.

According to these parameters, a limit on the detectability of the tracer will control the minimum affordable $K b_{0}$ valuc. The $K E D_{50}$ value along with the steepness of the doseresponse curve will then determine the response properties of the assay. In practice, random errors associated with measurement of low levels of bound enzyme-labeled species, pipetting errors, phase separation errors, etc., will statistically determine the detection capabilities of the assays (9,27-29). Such statistical constraints can be evaluated if the response can be fitted to a four-parameter logistic model as suggested by Dudley et al. (30). However, this approach cannot be used to predict the absolute effect of reagent concentrations on the characteristics of the dose-response curves. Halfman and Schneider $(28,29)$ have suggested optimization methods which include such statistical constraints (based on 
a mass-action model) but their approach is restricted to the simple case of $K^{*}=K$, which is even further simplified by assuming that the binder and the analyte are present in the assay system at concentrations greater than $1 / K$ and that $p^{*}>q$. The more general approach of Yanagishita and Rodbard (31) is restricted to the case of equal affinity of the labeled and unlabeled ligand toward the binder. Consequently, statistical restrictions are not considered here because for our model there is no simple analytical solution for the variance on the dose-response curve. However, in order to suggest reagent concentrations to be used when bridging group recognition occurs, assumptions regarding the minimum amount bound at zero dose, $b_{0}$, are required, as is discussed later.

In equilibrium type methods, the difference in the affinity constants of the unlabeled and enzyme-labeled ligand for the binder drastically affects the performance of the assays. This effect is summarized in Fig. 1 for values of binder and label concentrations in the range typically found in immunoassays (i.e., $K q$ $=50, K p^{*}=50$ ). In this case and in all subsequent studies the parameters $K q$ and $K p^{*}$ refer to concentrations of the species in the assay tube. The same is true for the parameters $K b_{0}, \mathrm{KED}_{50}$, steepness, and relative steepness. Curves gencrated with $K^{*} / K$ ratios of 10 and 100 correspond to the case of relatively higher affinity constants for the conjugated than for the unlabeled ligand, a situation that is often encountered when there is bridging group recognition.

To better predict the properties of the equilibrium assay system for changes in the concentration of the reagents, parametric studies were undertaken. The effects of varying the ratio $p^{*} / q$ on $\mathrm{KED}_{50}$ and the steepness of the dose-response curve for given $K q$ (e.g., $K q$ $=50$ ) are shown in Figs. 2 and 3. Figure 2 indicates that for given $K q$ and $K p^{*}$ values. the lower the $K^{*} / K$, the lower the $\mathrm{KED}_{50}$ value and, potentially, the lower the concentration of unlabeled ligand that can be detected. The actual detection limits, however, will be de-

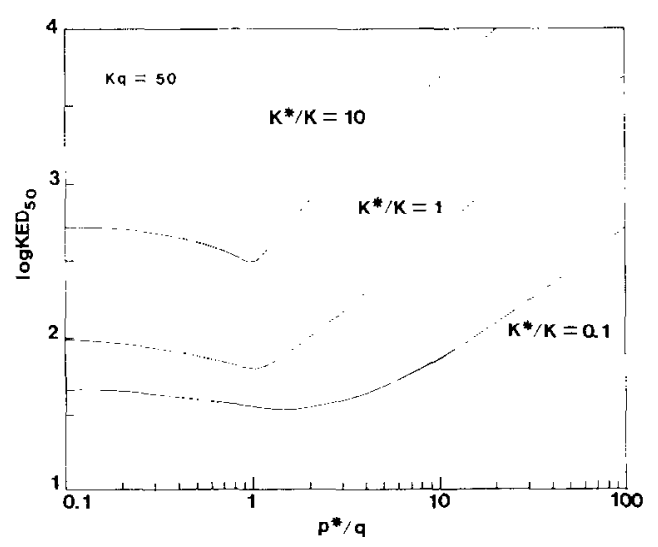

FIG. 2. Effect of changes in the $p^{*} / q$ ratio (for $K q=50$ ) on the $\log \mathrm{KED}_{50}$ value of equilibrium mode assays for various $K^{*} / K$ ratios.

termined by several factors, including the detectability of the enzyme-labeled ligand. As can also be seen in Fig. 2, a minimum value of $\mathrm{KED}_{50}$ is always observed at or near $p^{*} / q=1$. The increase in $\mathrm{KED}_{50}$ is marked as $p^{*} / q$ increases above this minimum, while the rate of increase is slower as $p^{*} / q$ decreases below the minimum. From Fig. 3 it is evident that there is an optimum relationship between $K^{*}$ and $K$ for maximum relative steepness and for given $K q$ and $K p^{*}$ values. Indeed, the relative steepness of the dose-response curve is reduced drastically at very high or very low values of $K^{*}$ relative to $K$. However, it can also be seen that bridging group recognition has less of an effect on the relative steepness of dose-response curves as the labeled ligand concentration approaches or exceeds the binding site concentration.

The effect of combined changes in reagent concentration $\left(K q\right.$ and $\left.K p^{*}\right)$ on the theoretical model for $K^{*} / K=10$ (bridging group recognition) is illustrated in Fig. 4. In most instances, the use of a lower concentration of binder and/or enzyme-labeled ligand will decrease the $\mathrm{KED}_{50}$ value, but at the same time decrease the steepness of the curve. The shape of the $\mathrm{KED}_{50}$ contour, at regions where $p^{*}>q$ and $q>p^{*}$, is almost perpendicular to the $K p^{*}$ and $K q$ axes, respectively. Thus, in order to improve the $\mathrm{KED}_{50}$ values when $p^{*}>q$, a 


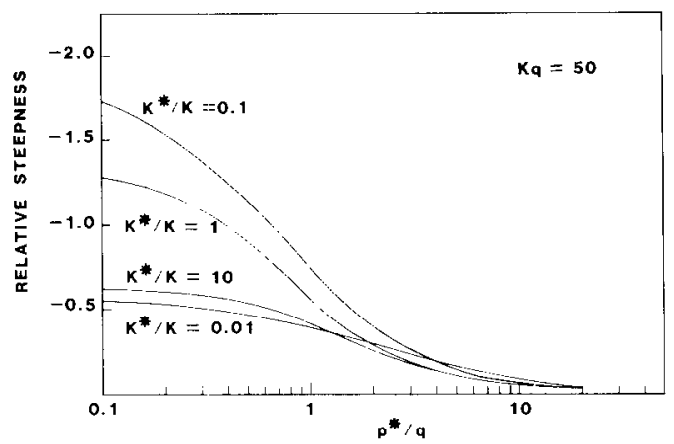

FIG. 3. Relationship between relative steepness of doseresponse curves and $p^{*} / q$ for $K q=50$ and various $K^{*} / K^{*}$ ratios (equilibrium mode).

decrease in $K p^{*}$ is necessary while a change in $K q$ does little to reduce the $\mathrm{KED}_{50}$ value. Equivalently, when $q>p^{*}$, a decrease in $q$, not changes in $p^{*}$, is desired to improve the $\mathrm{KED}_{50}$ value. From this figure it can also be inferred that increasing the volume of the incubation mixture, which is equivalent to simultaneously decreasing the concentrations of both $q$ and $p^{*}$, will improve the $\mathrm{ED}_{50}$ but at the expense of the steepness of the dose-response curve. The same general conclusions can be drawn for the case where $K^{*} / K=1$ (Fig. 5). However, when there is no bridging

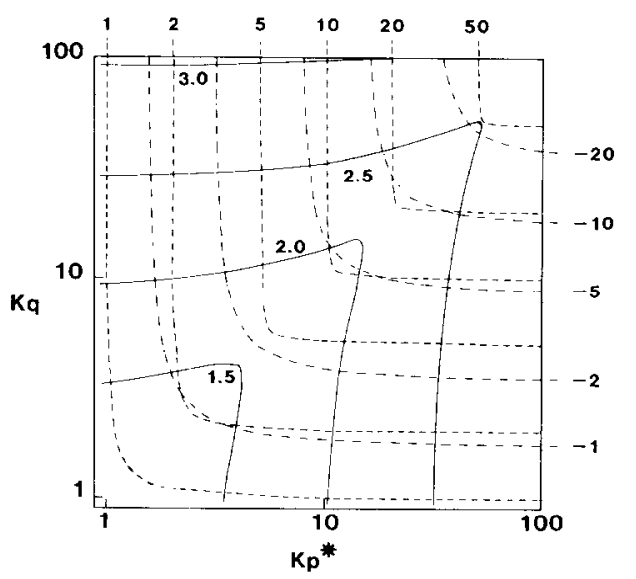

FIG. 4. Contours of $\log \mathrm{KED}_{50}$ values $(-; 1.5$ to 3.0 within figure borders), steepness $(-\cdot-\cdot-;-1$ to -20 on right margin), and $K b_{0}$ (---: 1 to 50 on top margin) evaluated for $K^{*} / K=10$ and combined changes in $K_{q} q$ and $\kappa p^{*}$ (equilibrium mode).

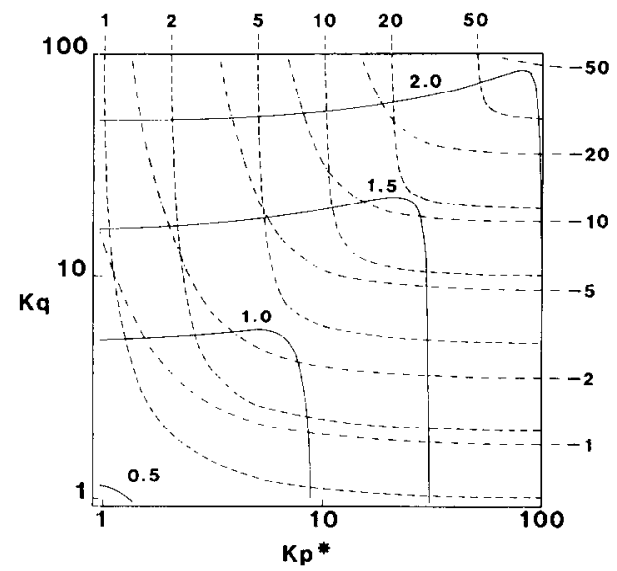

FIG. 5. A family of contours for $\log \mathrm{KED}_{50}$ values (0.5 to 2.0 within figure borders), steepness $(-\cdot-\cdot-;-1$ to -50 on right margin), and $K h_{0}$ (---; 1 to 50 on top margin) evaluated for $K^{*} / K=1$ and combined changes in $K q$ and $\kappa p^{*}$ (equilibrium mode).

group recognition there is almost a 10 -fold improvement in $\mathrm{KED}_{50}$ values for the same $K p^{*}$ and $K q$ values shown in Fig. 4. There is less of an effect on steepness and $K b_{0}$ values. It should be noted that the regions examined on Figs. 4 and 5 are only those which would be useful for analytical purposes.

An optimized assay protocol should employ an enzyme conjugate concentration, $p^{*}$, so that its enzymatic activity can be easily monitored within a reasonably short measuring period. Therefore, it is critical to employ an enzyme conjugate with high specific enzymatic activity for which sensitive detection techniques are available (i.e., fluorescence). To demonstrate the utility of Figs. 4 and 5 in optimizing assays, a hypothetical assay to measure an analyte with a desired $\mathrm{ED}_{50}$ value of $2 \times 10^{-9} \mathrm{M}$ is presented (if $100 \mu \mathrm{l}$ of sample is used in a total assay volume of $1 \mathrm{ml}$, the corresponding $\mathrm{ED}_{50}$ for the sample will be 2 $\times 10^{-10} \mathrm{M}$ ). This assay assumes an antibody association constant toward the unlabeled ligand of $5 \times 10^{10} \mathrm{M}^{-1}\left(\log \mathrm{KED}_{50}=2\right)$ and a $K^{*} / K$ ratio of 10 . A further assumption is that the concentration of the enzyme conjugate bound to the immobilized binder is high enough so that a sufficient absorbance signal 
can be measured in the zero dose tube over a relative short period of time (e.g., $30 \mathrm{~min}$ ). This $b_{0}$ value is highly dependent on the molecular weight and the specific activity of the enzyme label. For this example, a value of 2 $\times 10^{-11} \mathrm{M}$ was assumed as a minimum requirement for $b_{0}\left(K b_{0}=1\right)$. In the case of alkaline phosphatase, a label commonly used in enzyme immunoassays, this $b_{0}$ corresponds to an absorbance signal of 0.666 after $30 \mathrm{~min}$ incubation of the enzyme with the substrate (absorbance of sample minus the absorbance of the blank) assuming a molecular weight of 100,000 (32) and a specific activity of 500 units/mg for the enzyme (the product of the enzymatic reaction, $p$-nitrophenol, has an $\epsilon_{405}$ $=18.5 \times 10^{3} \mathrm{M}^{-1} \mathrm{~cm}^{-1}$ ). Based on these assumptions, to optimize a dose-response curve to attain given $\mathrm{ED}_{50}$ and required minimum $b_{0}$ values, the reagent $p^{*}$ and $q$ concentrations can be easily found from Fig. 4 as the points on the $\log \mathrm{KED}_{50}$ contour that give values of $K b_{0} \geqq 1$. Since, in general, the desired two contours intersect at two points, two solutions are possible for each specific requirement for $\mathrm{ED}_{50}$ and $b_{0}$ values. The intersect that gives $p^{*}>q$ is preferred since this conserves binder and at the same time yields dose-response curves with steepnesses similar to the alternate solution, when the curves are plotted as bound labeled vs log dose. However, if the response curves are examined as bound labeled vs dose (rectangular coordinates), it is evident that the first set of reagent concentrations also results in curves with better initial slopes compared to the solution when $p^{*}<q$, and therefore, lower concentrations of analyte may be determined.

From Fig. 4, the concentrations of the reagents that will yield the desired assay response curve using the minimum amount of binder possible are $2.08 \times 10^{-10} \mathrm{M}$ for $p^{*}$ and 2.02 $\times 10^{-11} \mathrm{M}$ for $q$. Note that the steepness (absolute) of the curves can be improved by increasing $p^{*}$ and/or $q$ on the given $\mathrm{ED}_{50}$ contour above these two intersection points. Such steepness is associated with higher $b_{0}$ values which may permit a lower incubation time of the enzyme with the substrate(s), and thus, faster assays. For example, the dose-response curve that corresponds to $K q=2$ and $K p^{*}$ $=10$ will have a $K b_{0}$ value of 1.98 . The almost twice as high $K b_{0}$ value (compared to the one obtained when $K q=1.01$ and $K p^{*}=10.4$ ) allows the use of a $15-\mathrm{min}$, rather than a 30 min, incubation time period to generate the same absorbance signal of 0.666 . However, if the $b_{0}$ value is too high, problems may occur because either the incubation time would need to be too short (irreproducible results may thus be obtained) or the absorbance would be too high to be measured accurately. In comparison, for the same $p^{*}$ and $q$, if no bridging group recognition were to occur $\left(K^{*} / K=1\right)$, a lower $E D_{50}$ value would be obtained without substantial reduction of $b_{0}$ (see Fig. 5).

The effect of the multivalency of the conjugate on the relative steepness and $\mathrm{KED}_{50}$ values of dose-response curves is shown in Fig. 6 for initial $K^{*} / K$ ratios of 10,1 , and 0.1 . As noted earlier, increasing the number of ligands attached to the enzyme label effectively causes $K^{*}$ to become $n K^{*}=K_{\text {stat }}$, where $n$ is the number of ligands attached to sites capable of being bound by the immobilized binder. This model applies when one assumes that once a ligand is bound by the binder, other ligands on the enzyme cannot also bind to the solid

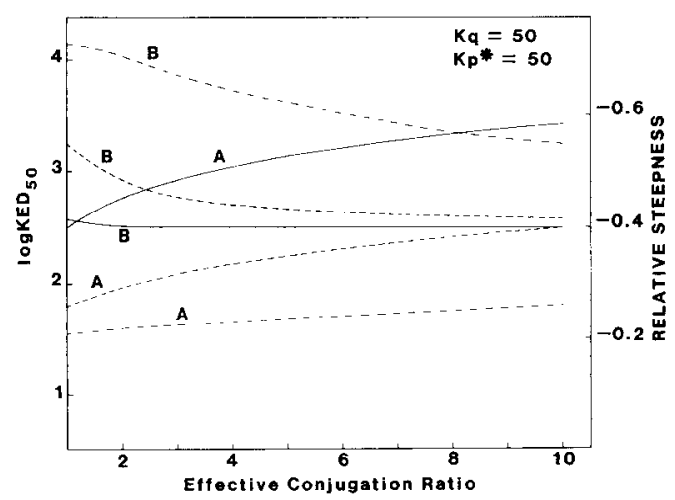

FIG. 6. Effect of conjugate substitution on $\log \mathrm{KED}_{50}$ (curves A) and relative steepness (curves B) for three initial $K^{*} / K$ values: $K^{*} / K=10(-), K^{*} / K=1(--)$, and $K^{*} /$ $K=0.1(-\cdot \cdot-)$. These curves were generated for equilibrium assays and $K q=K p^{*}=50$. 
phase. This assumption may or may not be true, but serves as a conservative model since, if multiple ligands attached to the same enzyme could be bound simultaneously this would increase $K_{\text {stat }}$ enormously (up to $10^{4}$ times, Ref. (33)) and make bridging group recognition problems even worse. It is evident from Fig. 6 that at least in the case of an enzyme conjugate that possesses a chemical bridge recognizable by the binder $\left(K^{*} / K>1\right)$, the use of a multivalent conjugate will worsen the detection capabilities of the assay. This theoretical finding is in agreement with experimental data $(11,34)$.

From the above discussions, whenever $K^{*}$ $>K$, detection capabilities of the immunoassays, in particular EIAs, deteriorate. Several techniques have been used in practice to overcome this bridging group recognition problem. For example, Van Weemen and Schuurs (4) first realized the importance of heterology in their EIA for estrogens. Improved detection limits were abtained when the estrogen derivatives employed for the preparation of the immunogen and the enzyme conjugate were structurally related but different than the analyte. By employing such a technique, the affinity constant between the antibody and enzyme-labeled hapten is reduced without any change in the affinity between the binder and the unlabeled hapten. Thus, the unlabcled hapten has a better chance to compete with the conjugate for the binding sites of the antibody. However, structurally similar haptens may become greater interferents in the assay. Indeed. Meyerhoff and Rechnitz $(35,36)$ used cyclic GMP-enzyme conjugate and anti-cyclic AMP antibody to assay lower levels of cyclic AMP. This approach, however, resulted in reduced selectivity over cyclic GMP.

Similarly, Van Weemen and Schuurs $(5,6)$ investigated in detail the properties of heterologous assays. Besides hapten heterology, they studied the effect of changing the nature of the bridging group used in the preparation of the enzyme-hapten conjugate (bridge heterology) and altering the site of hapten attachment to the enzyme (site heterology). Finally, a com- bination of two or more of the heterology types is also possible (5). In all their studies such approaches resulted in improved detection limits of the assays but at the expense of reduced selectivity.

This technique of lowering the binding affinity between the enzyme-labeled species and the binder has been modeled for the case of $K q=K p^{*}=50$ and for $K / K_{\mathrm{I}}=100$, where $K_{1}$ is the association constant of the binder with the interferent (Fig. 7). A heterologous assay will reduce $K^{*}$ without affecting the $K$ and $K_{\mathrm{I}}$. As shown, a reduction in the $K^{*}$ will improve the detection capabilities toward the analyte as reflected by the $\mathrm{KED}_{50}$ values but at the same time, the selectivity $\left(\mathrm{ID}_{50} / \mathrm{ED}_{50} ; \mathrm{ID}_{50}\right.$ equals the effective dose of the interferent that produces $50 \%$ of the maximum response) deteriorates substantially (i.e., increased crossreactivity).

A second approach for improving enzymelinked competitive binding assays has recently been suggested by this research team $(17,18)$. This approach involves the use of endogenous binding proteins rather than antibodies. These binding proteins are either specific globular protein molecules with binding sites for a particular ligand (e.g., serve to transport ligands within the body) or they may be certain en-

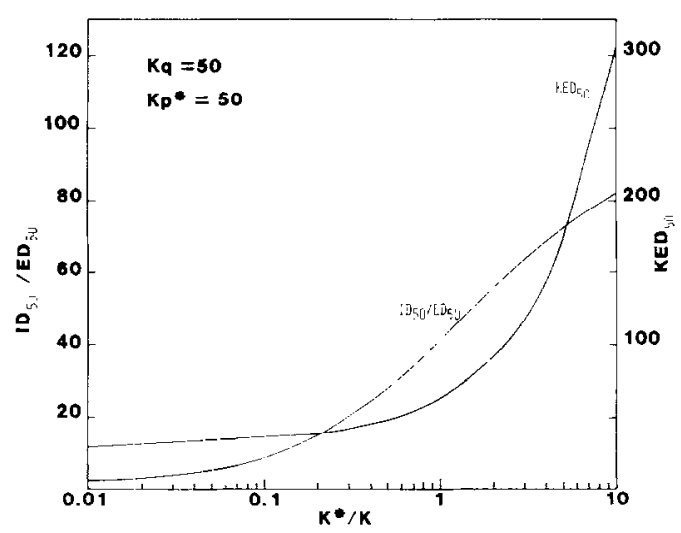

FIG. 7. Predicted $\mathrm{KED}_{50}$ values (for analyte) and interferent selectivity, $\mathrm{ID}_{50} / \mathrm{ED}_{50}$, in heterologous equilibrium asays. Curves were generated assuming different degrees of heterology $\left(K^{*} / K^{\prime}\right.$ ratio $)$ and $K / K_{I}=100\left(K q\right.$ and $K p^{*}$ were fixed at 50 ). 
zymes which possess allosteric ligand binding sites. Since the binder production need not be elicited by immunological procedures, there should be a naturally similar or higher affinity for the unlabeled than for the enzyme-labeled ligands (i.e., $K^{*} / K \leqq 1$ ). As shown in Figs. 2, and 3 this situation gives rise to improved steepness and lower $\mathrm{KED}_{50}$ values. Such assays involving the use of appropriate binding proteins (immobilized on a solid phase support) in conjunction with a ligand-enzyme conjugate have been developed for the detection of folate, vitamin $B_{12}$, and thyroxine $(17,18)$. Naturally, such binding protein systems can be utilized only if they bind the analyte and the enzyme-labeled ligand with the high affinity necessary to detect low levels of analyte. Further, the selectivily obtained with such systems is controlled by the natural affinities of the binding protein toward the analyte and the respective interferents.

A third approach to solve the bridging group recognition problem is to perform assays using a sequential binding technique. Such a technique is dependent on kinetics rather than thermodynamics and is controlled by two sets of association and dissociation rate constants (see above model).

A collection of values of association rate constants (37) for polyclonal antibodies with various radiolabeled haptens indicated relatively small differences. Values fell roughly in the 10 -fold range of $10^{7}$ to $10^{8} \mathrm{M}^{-1} \mathrm{~s}^{-1}\left(10^{8}\right.$ $\mathrm{M}^{-1} \mathrm{~s}^{-1}$ is the proposed diffusion controlled limit for this rate constant if the electrostatic repulsions between the hapten and the antibody are taken into account (38)), while the dissociation rate constants varied in a 1000 fold range. Smith and Skubitz (39) observed that the dissociation rate constants corresponding to the kinetics of the hapten with its respective hapten-specific antibody were lower than those corresponding to the same antibody with a hapten similar in structure but different than the one used to induce the antibody production. For example, rabbit digitoxin specific antibody had association rate constants of 1.4 $\times 10^{7}, 1.1 \times 10^{7}$, and $1.3 \times 10^{7} \mathrm{M}^{-1} \mathrm{~s}^{-1}$ with $\left[{ }^{3} \mathrm{H}\right]$ digitoxin, $\left[{ }^{3} \mathrm{H}\right]$ digoxin, and $\left[{ }^{3} \mathrm{H}\right]$ ouabain, respectively. On the other hand, the respective dissociation rate constants were $2.3 \times 10^{-4}$, $1.2 \times 10^{-3}$, and $1.4 \times 10^{-2} \mathrm{~s}^{-1}$.

Generally, in sequential binding methods the first incubation is carried to equilibrium. Dose-response curves for this case are shown in Fig. 8 where the second incubation time is varied for fixed $K^{*} / K$ and $k_{-2} / k_{-1}$ ratios. This variation is equivalent to variation in $k_{-1} t_{2}$ values. The unitless parameter $k_{-1} t_{2}$ is used so that either differences in the second incubation time or differences in the dissociation rates of the unlabeled species can be assessed. As expected, an increase in the second incubation time will result in dose-response curves that will eventually approach those obtained using an equilibrium type of assay. A value of $k_{-1} t_{2}$ $=0.1$ is long enough for the label to nearly saturate available binding sites, but short enough so that significant reequilibration does not occur. This value will be used to generate all subsequent sequential binding assay data.

The dose-response curves presented in Fig. 9 were simulated using the parameters given in Table 1. Curves $A_{1}, A_{2}$, and $A_{3}$ were generated using a $K^{*} / K$ ratio of 10 and various $k_{-2} / k_{-1}$ values. Similarly, curves $\mathbf{B}_{1}, \mathbf{B}_{2}$, and

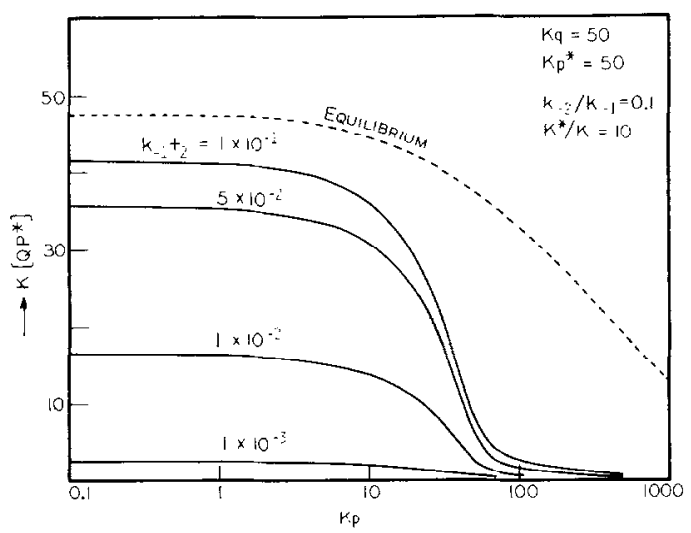

FIG, 8. Dose-response curves of a sequential binding assay for $K^{*} / K=10$ and given $K q$ and $K p^{*}$. The first incubation time was considered long enough to allow equilibrium while the second one was varied as indicated by the $k_{-1} t_{2}$ parameter. An equilibrium assay curve was generated using same parameters. 


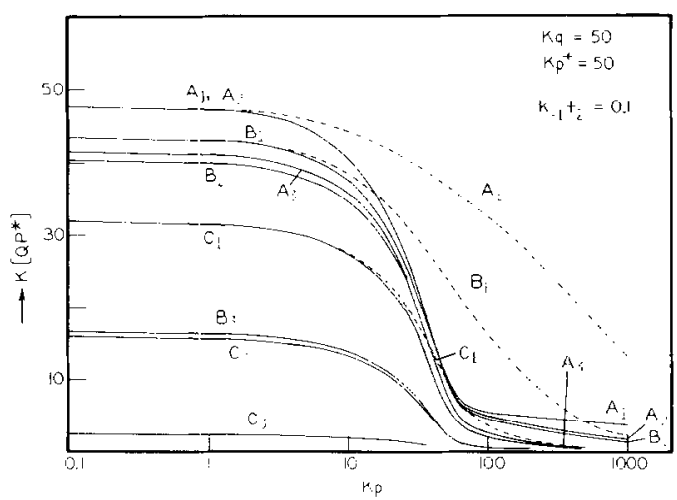

FIG. 9. Sequential binding dose-response curves with $K^{*} / K$ and $k_{-2} / k_{-1}$ ratios as shown in Table 1 (fixed $K q$ and $K p^{*}$ values at 50 and fixed second incubation time $t_{2}$ so that $\left.k_{-1} l_{2}=0.1\right)$. Labels A, B, and C refer to decreasing ratios of $K^{*} / K(10,1,0.1)$. Numerals 1 through 3 represent the ratios $k_{-2} / k_{-1}\left(10,1,0.1\right.$, respectively). Cases $A_{E}, B_{E}$, and $C_{E}$ denote the corresponding equilibrium mode assays.

$\mathrm{B}_{3}$ correspond to a $K^{*} / K$ value of 1 , and curves $\mathrm{C}_{1}, \mathrm{C}_{2}$, and $\mathrm{C}_{3}$ to a $K^{*} / K$ ratio of 0.1 . In all cases it can be seen that the lower the $k_{2} / k_{1}$ ratio, the shallower the curve and the lower the $k b_{0}$ value. Further, the same trend is evident when the $K^{*} / K$ ratio is lowered for a given $k_{-2} / k_{-1}$ ratio. In addition, in the case of low $K^{*} / K$ (e.g., equal to 0.1 and $k_{-2} / k_{-1}$ equal to 10$)$ there was almost no advantage to sequential binding mode over the equilibrium mode assays.

An obvious disadvantage of this type of assay protocol is that it involves a second incubation period which must be accurately controlled, thus increasing the time and difficulty of the assay. Further, sequential binding type assays suffer a dramatic deterioration in their selectivity characteristics. For example, $E D_{50}$ values of dose-response curves for the analyte and the ratio $\mathrm{ID}_{50} / \mathrm{ED}_{50}$ for the interferent are shown in Table 2 along with the parameters used to generate these curves. In Table 2 it is assumed that all differences in the affinity constants come from differences in the dissociation rate constants while the association rate constants remain unaltered. Based on known data $(37,39)$ this is a reasonable assumption although it may not be valid in every case. The $\mathrm{ED}_{50}$ values for the analyte and the interferent indicate that in all three cases of Table 2 there is very limited selectivity $\left(\mathrm{ID}_{50}\right.$ values very close to the corresponding $\mathrm{ED}_{50}$ values) of the assays. This theoretical finding has also been observed experimentally and was empirically explained by a "first come, first served" mechanism (40,41). Thus, unless it is desired to measure a whole class of compounds this mode of assay should be avoided.

In summary, several conclusions can be drawn from the models presented here. For hapten EIAs in which the antibody exhibits bridging group recognition toward the enzyme-labeled species, equilibrium type assays using analyte-enzyme conjugates with the same bridge as the immunogen will result in assays with limited detection capabilities, as defined by high $\mathrm{ED}_{50}$ values and a lower steepness for the dose-response curves. In such cases, theory predicts that assay performance can be optimized to lower $\mathrm{ED}_{50}$ values by varying the concentrations of binder and/or labeled ligand; however, this will reduce the steepness of the dose-response curve and, concomitantly, the precision of the method. Further, for given $p^{*}$ or $q$ concentrations, steeper dose-response curves with lower $\mathrm{ED}_{50}$

\section{TABLE 1}

Paramliters Used to Model Sequential Binding DOSE-RESPONSE CuRves ShOWN IN FiG. 9

\begin{tabular}{cccc}
\hline Curve & $K^{*} / K$ & $k_{-2} / k_{-1}$ & $k_{2} / k_{1}$ \\
\hline $\mathrm{A}_{1}$ & 10 & 10 & 100 \\
$\mathrm{~A}_{2}$ & 10 & 1 & 10 \\
$\mathrm{~A}_{3}$ & 10 & 0.1 & 1 \\
$\wedge_{\mathrm{E}}$ & 10 & $-a$ & - \\
$\mathrm{B}_{1}$ & 1 & 10 & 10 \\
$\mathrm{~B}_{2}$ & 1 & 1 & 1 \\
$\mathrm{~B}_{3}$ & 1 & 0.1 & 0.1 \\
$\mathrm{~B}_{\mathrm{E}}$ & 1 & - & - \\
$\mathrm{C}_{1}$ & 0.1 & 10 & 1 \\
$\mathrm{C}_{2}$ & 0.1 & 1 & 0.1 \\
$\mathrm{C}_{3}$ & 0.1 & 0.1 & - \\
$\mathrm{C}_{\mathrm{E}}$ & 0.1 & - & 0.01 \\
\hline
\end{tabular}

${ }^{a}$ Rate constants not considered in equilibrium mode assays. 
TABLE 2

Parameters Used and Results Obtained for Sequential Binding Interferencf Study

\begin{tabular}{lccccc}
\hline \multicolumn{1}{c}{ Curve } & $K^{*} / K$ & $k_{-2} / k_{-1}$ & $k_{2} / k_{1}$ & $\mathrm{KED}_{50}$ & $\mathrm{ID}_{50} / \mathrm{ED}_{50}$ \\
\hline Analyte & 10 & 0.1 & 1 & 31.2 & 1.00 \\
Interferent & 1000 & 0.001 & 1 & & \\
Analyte & 1 & 1 & 1 & 31.4 & 1.01 \\
Interferent & 100 & 0.01 & 1 & 31.9 & 0.98 \\
Analyte & 0.1 & 10 & 1 & & \\
Interferent & 10 & 0.1 & 1 & & \\
\hline
\end{tabular}

values are obtained at $p^{*} \simeq q$ (Figs. $2,4,5$ ). In addition, the models presented suggest the use of conjugates with low hapten to enzyme ratio to avoid compounding the bridging group recognition problem. Equilibrium assays utilizing heterologous conjugates (i.e., different bridging group) may improve the performance of assays, but if the degree of heterology is too excessive, assays with highly reduced selectivity will result. Performing sequential binding type assays will, generally, increase the steepness and lower the $E_{50}$ value of the dose-response curve but with a concomitant severe loss of assay selectivity. The concept of using endogenous hapten selective binding proteins rather than antibodies appears to offer a promising new approach since bridging group recognition is completely circumvented. However, such an approach is limited to systems for which there exists a suitable binding protein which will have a high enough affinity toward the analyte and enzyme-labeled conjugate so that competitive binding assays with detection capabilities comparable to traditional immunoassays (e.g., RIAs) will result.

Finally, while this study was undertaken to help explain and predict observations in hapten EIA systems, the models and data presented here should help workers developing other types of immunoassays (e.g., fluoroimmunoassays and RIAs) to fully optimize the performance of their tests.

\section{APPENDIX I}

\section{Mass Action Model of Heterogeneous Enzyme-Linked Assays}

To predict the effect of the difference between $K$ and $K^{*}$ on heterogeneous enzymelinked competitive binding assays, dose-response curves were simulated assuming a single-incubation equilibrium system which employs a binder possessing univalent, independent, and equivalent binding sites and a univalent conjugate. These assumptions lead to the mass action model

$$
\begin{gathered}
\mathrm{P}+\mathrm{Q} \rightleftharpoons \mathrm{QP}, \quad K \\
\mathrm{P}^{*}+\mathrm{Q} \rightleftharpoons \mathrm{QP}^{*}, \quad K^{*}
\end{gathered}
$$

where $\mathrm{P}$ is the unlabeled ligand, $\mathrm{P}^{*}$ is the ligand-enzyme conjugate, and $Q$ is the specific binder. $K$ and $K^{*}$ are the association constants for the two reactions. The initial concentrations of $\mathrm{P}, \mathrm{P} *$, and $\mathrm{Q}$ are symbolized by $p, p^{*}$. and $q$, respectively.

For simplification purposes, the incubation time will be considered long enough to reach equilibrium. Then, the following mass action equations can be written:

$$
\begin{aligned}
K & =\frac{[\mathrm{QP}]}{[\mathrm{P}][\mathrm{Q}]} \\
K^{*} & =\frac{\left[\mathrm{QP}^{*}\right]}{\left[\mathrm{P}^{*}\right][\mathrm{Q}]}
\end{aligned}
$$




$$
\begin{aligned}
p & =[\mathrm{P}]+[\mathrm{QP}] \\
q & =[\mathrm{Q}]+[\mathrm{QP}]+\left[\mathrm{QP}^{*}\right] \\
p^{*} & =\left[\mathrm{P}^{*}\right]+\left[\mathrm{QP}^{*}\right] .
\end{aligned}
$$

Equations [1] and [2] are solved for [QP] and $\left[\mathrm{QP}^{*}\right]$, respectively, taking into account the values of $[\mathrm{P}]$ and $\left[\mathrm{P}^{*}\right]$ from [3] and [5].

$$
\begin{aligned}
{[\mathrm{QP}] } & =\frac{K p[\mathrm{Q}]}{1+K[\mathrm{Q}]} \\
{\left[\mathrm{QP}^{*}\right] } & =\frac{K^{*} p^{*}[\mathrm{Q}]}{1+K^{*}[\mathrm{Q}]}
\end{aligned}
$$

Then, by substitution into [4]

$$
q=[\mathrm{Q}]+\frac{K p[\mathrm{Q}]}{1+K[\mathrm{Q}]}+\frac{K^{*} p^{*}[\mathrm{Q}]}{1+K^{*}[\mathrm{Q}]}
$$

or equivalently (normalized with respect to $K$ )

$$
\begin{aligned}
& K q=K[\mathrm{Q}]+\frac{K p K[\mathrm{Q}]}{1+K[\mathrm{Q}]} \\
&+\frac{\left(K^{*} / K\right) K p^{*} K[\mathrm{Q}]}{1+\left(K^{*} / K\right) K[\mathrm{Q}]} .
\end{aligned}
$$

Given $K p, K p^{*}, K q$, and $K^{*} / K$ this equation can be solved for $K[Q]$ by the secant numerical approximation method (42). Once $K[\mathrm{Q}]$ is known, $K\left[\mathrm{QP}^{*}\right]$ can be calculated from the normalized (with respect to $K$ ) version of $\mathrm{Eq}$. [7]:

$$
K\left[\mathrm{QP}^{*}\right]=\frac{\left(K^{*} / K\right) K p^{*} K[\mathrm{Q}]}{1+\left(K^{*} / K\right) K[\mathrm{Q}]}
$$

\section{APPENDIX $\|$}

\section{Mass Action Model for the Sequential Binding Assay}

The sequential binding assay involves two incubation steps. First, the unlabeled ligand is incubated with the binder for a period of time which may or may not be enough to allow complete equilibrium:

$$
\mathbf{P}+\mathrm{Q} \underset{k-1}{\stackrel{k_{1}}{\rightleftharpoons}} \mathrm{QP}, \quad K=k_{1} / k_{-1} .
$$

This preincubation step is described by the following set of equations:

$$
\begin{aligned}
\frac{d[\mathrm{QP}]}{d t} & =-k_{-1}[\mathrm{QP}]+k_{\mathrm{I}}[\mathrm{P}][\mathrm{Q}] \\
p & =[\mathrm{P}]+[\mathrm{QP}] \\
q & =[\mathrm{Q}]+[\mathrm{QP}]
\end{aligned}
$$

The labeled ligand is then added to the reaction mixture followed by a second incubation:

$$
\begin{gathered}
\mathrm{P}+\mathrm{Q} \underset{k_{-1}}{\stackrel{k_{1}}{\rightleftharpoons}} \mathrm{QP}, \quad K=k_{1} / k_{-1} \\
\mathrm{P}^{*}+\mathrm{Q} \underset{k_{-2}}{\stackrel{k_{2}}{\rightleftharpoons}} \mathrm{QP}^{*}, \quad K^{*}=k_{2} / k_{-2} .
\end{gathered}
$$

The parameters of this incubation step are described by the equations

$$
\begin{aligned}
\frac{d[\mathrm{QP}]}{d t} & =-k_{-1}[\mathrm{QP}]+k_{1}[\mathrm{P}][\mathrm{Q}] \\
\frac{d\left[\mathrm{QP}^{*}\right]}{d t} & =-k_{-2}\left[\mathrm{QP}^{*}\right]+k_{2}\left[\mathrm{P}^{*}\right][\mathrm{Q}] \\
p & =[\mathrm{P}]+[\mathrm{QP}] \\
q & =[\mathrm{Q}]+[\mathrm{QP}]+\left[\mathrm{QP}^{*}\right] \\
p^{*} & =\left[\mathrm{P}^{*}\right]+\left[\mathrm{QP}^{*}\right] .
\end{aligned}
$$

Although analytical solutions of this systcm are available for the case where $k_{1}=k_{2}$ and $k_{-1}=k_{-2}(43,44)$, the system gets quite complicated in the general case of $k_{1} \neq k_{2}$ and $k_{-1}$ $\neq k_{-2}$. Therefore, a numerical solution is necessary. Because of its simplicity, the rectangular rule for numerical integration was employed to solve the differential equations of this model (although better but more complex methods do exist, e.g., Runge-Kutta integration). The two differential equations can then be rewritten as

$$
\begin{gathered}
\Delta[\mathrm{QP}]=\Delta t\left(-k_{-1}[\mathrm{QP}]+k_{1}[\mathrm{P}][\mathrm{Q}]\right) \\
\Delta\left[\mathrm{QP}^{*}\right]=\Delta t\left(-k_{-2}\left[\mathrm{QP}^{*}\right]+k_{2}\left[\mathrm{P}^{*}\right][\mathrm{Q}]\right)
\end{gathered}
$$

or

$$
\begin{aligned}
& K[\mathrm{QP}]_{n}-K[\mathrm{QP}]_{n-1} \\
& \quad=-k_{-1} \Delta t K[\mathrm{QP}]_{n-1}+k_{-1} \Delta t K[\mathrm{P}]_{n-1} K[\mathrm{Q}]_{n-1}
\end{aligned}
$$




$$
\begin{aligned}
& K\left[\mathrm{QP}^{*}\right]_{n}-K\left[\mathrm{QP}^{*}\right]_{n-1} \\
& \quad=-\left(k_{-2} / k_{-1}\right) k_{-1} \Delta t K\left[\mathrm{QP}^{*}\right]_{n-1} \\
& \quad+\left(k_{-2} / k_{-1}\right) k_{-1} \Delta t\left(K^{*} / K\right) K\left[\mathrm{P}^{*}\right]_{n-1} K[\mathrm{Q}]_{n-1} .
\end{aligned}
$$

In the case when the first incubation is long enough and equilibrium is reached, the equation

$$
K=\frac{[\mathrm{QP}]}{[\mathrm{P}][\mathrm{Q}]}
$$

holds and can be rewritten as

$$
K=\frac{[\mathrm{QP}]}{(q-[\mathrm{QP}])(p-[\mathrm{QP}])} .
$$

This relationship yields a quadratic equation which is used to calculate $K[\mathrm{QP}]$ :

$$
(K[\mathrm{QP}])^{2}-(1+k p+k q)(K[\mathrm{QP}])
$$

$$
+K q K p=0 .
$$

After $K[\mathrm{QP}]$ is estimated, the parameters $K[\mathrm{P}]$ and $K[\mathrm{Q}]$ are extracted from

$$
\begin{aligned}
& K[\mathrm{P}]=K p-K[\mathrm{QP}] \\
& K[\mathrm{Q}]=K q-K[\mathrm{QP}] .
\end{aligned}
$$

Thus, the model will calculate the parameters $K[\mathrm{P}], K[\mathrm{QP}]$, and $K[\mathrm{Q}]$ for the first incubation, which will serve as initial values for the parameters of the second incubation.

The final values of the parameters are estimated from the second incubation employing an iteration step of $k_{-1} \Delta t_{2}=1 \times 10^{-5}$ to ensure accuracy (i.e., if a $k_{-1} t_{2}$ value of 0.1 is used, a total of $1 \times 10^{4}$ iterations will be undertaken) and successive iterations controlled by the equations

$$
\begin{aligned}
K[\mathrm{QP}]_{n}=K[\mathrm{QP}]_{n-1} & -k_{-1} \Delta t_{2} K[\mathrm{QP}]_{n-1} \\
& +k_{-1} \Delta t_{2} K[\mathrm{P}]_{n-1} K[\mathrm{Q}]_{n-1}
\end{aligned}
$$

$$
\begin{aligned}
K\left[\mathrm{QP}^{*}\right]_{n} & \\
= & K\left[\mathrm{QP}^{*}\right]_{n-1}-\left(k_{-2} / k_{-1}\right) k_{-1} \Delta t_{2} K\left[\mathrm{QP}^{*}\right]_{n-1} \\
+ & \left(k_{-2} / k_{-1}\right) k_{-1} \Delta t_{2}\left(K^{*} / K\right) K\left[\mathrm{P}^{*}\right]_{n-1} K[\mathrm{Q}]_{n-1} \\
& K[\mathrm{P}]_{n}=K p-K[\mathrm{QP}]_{n}
\end{aligned}
$$

$$
\begin{aligned}
K\left[\mathrm{P}^{*}\right]_{n} & =K p^{*}-K\left[\mathrm{QP}^{*}\right]_{n} \\
K[\mathrm{Q}]_{n} & =K q-K[\mathrm{QP}]_{n}-K\left[\mathrm{QP}^{*}\right]_{n} .
\end{aligned}
$$

For every one of these iteration steps the parameters $K[\mathrm{QP}], K[\mathrm{P}]$, and $K[\mathrm{Q}]$ will be estimated.

This numerical integration procedure was verified for the limiting case of $k_{1}=k_{2}$ and $k_{-1}=k_{-2}$ by comparison to the integration method described by Rodbard et al. (24) and the analytical solution of DeLean and Rodbard (44).

\section{APPENDIX III}

\section{$K b_{0}, K E D_{50}$, Steepness, and Relative Steepness of Dose-Response Curves}

The $K b_{0}$ value is calculated from the described models by setting $K p=0$. To evaluate the $\mathrm{KED}_{50}$ for the equilibrium model, the value of $K b_{0} / 2$ is calculated first. Then, the $\mathrm{KED}_{50}$ is estimated numerically by the internal halving method as the value of $K p$ that corresponds to a $K\left[\mathrm{QP}^{*}\right]$ value of $K b_{0} / 2$. The $K E D_{50}$ in the case of sequential binding assays is determined graphically from the corresponding dose-response curves.

The steepness of a dose-response curve is defined as its maximum slope. This is equivalent to the maximum of the derivative:

$$
\frac{d\left(K\left[\mathrm{QP}^{*}\right]\right)}{d(\log K p)} \text {. }
$$

By taking the derivative of Eq. [6]

$$
\frac{d\left(K\left[\mathrm{QP}^{*}\right]\right)}{d(\log K p)}=\frac{K K^{*} p^{*}}{\left(1+K^{*}[\mathrm{Q}]\right)^{2}} \frac{d[\mathrm{Q}]}{d(\log K p)} .
$$

The derivative of Eq. [8] with respect to log $K p$ yields

$$
\frac{d[\mathrm{Q}]}{d(\log K p)}=-\frac{K p[\mathrm{Q}]\left(\log _{e} 10\right)}{(1+K[\mathrm{Q}]) A},
$$

where $A$ is given by the expression

$$
A=1+\frac{K p}{(1+K[\mathrm{Q}])^{2}}+\frac{K^{*} p^{*}}{\left(1+K^{*}[\mathrm{Q}]\right)^{2}} .
$$


Thus, the normalized equation (with respect to $K$ ) will be

$$
\begin{aligned}
\frac{d\left(K\left[\mathrm{QP}^{*}\right]\right)}{d(\log K p)} & = \\
& -\frac{K p K p^{*}\left(K^{*} / K\right) K[\mathrm{Q}]\left(\log _{e} 10\right)}{\left(1+\left(K^{*} / K\right) K[\mathrm{Q}]\right)^{2}(1+K[\mathrm{Q}]) A} .
\end{aligned}
$$

where $K[\mathrm{Q}]$ is calculated as described in Appendix I. The maximum of this function, i.e., the steepness of the dose-response curve, is calculated numerically by the golden section method (42).

The relative steepness is defined as the maximum of the derivative:

$$
\frac{d\left(\left[\mathrm{QP}^{*}\right] / p^{*}\right)}{d(\log K p)} \text {. }
$$

Following the same arguments as above

$$
\begin{aligned}
\frac{d\left(\left[\mathrm{Q}^{*}\right] / p^{*}\right)}{d(\log K p)} & = \\
& -\frac{K p\left(K^{*} / K\right) K[\mathrm{Q}]\left(\log _{e} 10\right)}{\left(1+\left(K^{*} / K\right) K[\mathrm{Q}]\right)^{2}(1+K[\mathrm{Q}]) A},
\end{aligned}
$$

which can be evaluated by the golden section method as above.

\section{REFERENCES}

1. Masseyeff, R. (1983) in Nuclear Medicine and Biology Advances, Proceedings World Congress, 3rd (Raynaud, C.. ed.), pp. 18-21, Pergamon, Oxford.

2. Blake, C. and Gould, B. J. (1984) Analyst 109, 533 547.

3. Monroe. D. (1984) Anal. Chem. 56, 920A-931A.

4. Van Weemen, B. K.. and Schuurs, A. H. W. M. (1972) FEBS Lett. 24, 77-81.

5. Van Weemen, B. K., and Schuurs, A. H. W. M. (1975) Immunochemistry' 12, 667-670.

6. Van Weemen, B. K. and Schuurs, A. H. W. M. (1976) in Immunoenzymatic Techniques (Feldman, G., Druet, P., Bignon, J.. and Avrameas, S., eds.), pp. 125-133, Elsevier, New York.

7. Sidki, A. M., Landon, J., and Rowell, F. (1984) Clin. Chem. 30, 1348-1352.

8. Knight, G. J., Wylie, P., Holman, M. S., and Haddow, J. E. (1985) Clin. Chem. 31, 118-121.

9. Jones. S. R., and Amatayakul, S. (1985) Clin. Chem. 31, 1076-1077.

10. Cameron. E. H. D., Scarisbrick, J. J., Morris, S. E., and Read, G. (1975) in Steroid Immunoassay (Cameron, E. H. D., Hillier, S. G., and Griffiths, K.. eds.), pp. 153-176, Alpha Omega, Cardiff.

11. Joyce, B. G., Read, G. F., and Riad-Fahmy, D. (1978) in Radioimmunoassay and Related Procedures in Medicine (Proc. Symp. Berlin, 1977). Vol. 1, pp. 289-295, IAEA, Vienna.

12. Nordblom, G. D., Webb, R., Counsell, R. E., and England, B. G. (1981) Steroids 38, 161-173.

13. Ekins, R. P., Newman, G. B., and O'Riordan, J. L. H. (1968) in Radioisotopes in Medicine: In Vilro Studies (Hayes, R. L., Goswiľ, F. A., and Murphy, B. E. P., eds.), pp. 59-100, U.S. Atomic Energy Commission, Oak Ridge, Tenn.

14. Feldman, H. A. (1972) Anal. Biochem. 48, 317-338.

15. Laurence, D. J., and Wilkison, G. (1974) Anal. Chem. 46, 1132-1135.

16. Naus, A. J., Kuppens, P. S., and Borst, A. (1977) Clin. Chem. 23, 1624-1627.

17. Bachas, L. G., Lewis, P. F., and Meyerhoff, M. E. (1984) Anal. Chem. 56, 1723-1726.

18. Bachas, L. G., Tsalta, C. D., and Meyerhoff, M. E. (1986) BioTechniques 4, 42-55.

19. Zettner, A. (1973) Clin. Chem. 19,699-705.

20. Zettner, A., and Duly, P. E. (1974) Clin. Chem. 20, $5-14$.

21. Greenwood, H. M., and Schneider, R. S. (1978) in Clinical Immunochemistry: Chemical and Cellular Bases and Applications in Disease (Natelson, S., Pesce, A. J., and Dietz, A. A., eds.), pp. 455-473, AACC, Washington, D.C.

22. Shaw, W., Hubert, I. L., Agnese, S. T., and Spierto, F. W. (1976) Clin. Chim. Acta 72, 69-82.

23. Rodbard, D. (1981) in Ligand Assay (Langan, J., and Clapp, J. J.. eds.), pp. 45-101. Masson, New York.

24. Rodbard, D.. Ruder, H. J., Vaitukaitis. J., and Jacobs, H. S. (1971) J. Clin. Endocrinol. 33, 343-355.

25. Ekins, R.. and Newman, B. (1970) Acta Endocrinol. Suppl. 147, 11-36.

26. Schuurs, A. II. W. M., and Van Weemen, B. K. (1977) Clin. Chim Acta 81, 1-40.

27. Rodbard, D. (1978) Anal. Biochem 90, 1-12.

28. Halfman, C. J. (1979) Anal. Chem. 51, 2306-2311.

29. Halfman. C. J., and Schneider, A. S. (1981) Anal. Chem. 53, 654-658.

30. Dudley, R. A., Edwards, P., Ekins, R. P., Finney, D. J., McKenzie, I. G. M., Raab. G. M., Rodbard, D.. and Rodgers, R. P. C. (1985) Clin. Chem. 31, 1264-1271.

31. Yanagishita, M., and Rodbard, D. (1978) Anal. Biochem. 88, 1-19.

32. Engstrom, L. (1961) Biochim. Biophy's Acta 52, 3648.

33. Gopalakrishnan, P. V.. and Karush. F. (1974) J. Immunol. 113, 769-778. 
34. Exley, D., and Abuknesha, R. (1978) FEBS Lett. 91, 162-165.

35. Meyerhoff, M. E., and Rechnitz, G. A. (1979) Anal. Biochem. 95, 483-493.

36. Meyerhoff, M. E., and Rechnitz, G. A. (1980) in Methods in Enzymology (Van Vunakis, H., and Langone, J. J., eds.), Vol. 70, pp. 439-454, Academic Press, New York.

37. Karush, F. (1978) Comp. Immunol. 5, 85-116.

38. Froese, A., and Sehon, A. H. (1965) Immunochemistry 2, 135-143.

39. Smith, T. W., and Skubitz. K. M. (1975) Biochemistry 14, 1496-1502.
40. Pratt, J. J., and Woldring, M. G. (1976) Clin. Chim. Acta 68, 87-90.

41. Vining, R. F., Compton, P., and McGinley, R. (1981) Clin. Chem. 27, 910-913.

42. Cheney. W., and Kincaid. D. (1980) Numerical Mathematics and Computing, Brooks/Cole, Monterey, Calif.

43. Rodbard, D., Munson, P. J.. and DeLean, A. (1978) in Radioimmunoassay and Related Procedures in Medicine (Proc. Symp. Berlin, 1977), Vol. 1, pp. 469-504, IAEA, Vienna.

44. DeLean, A., and Rodbard. D. (1978) in The Receptors (O’Brien, R. D., ed.), Vol. 1, pp. 43-192, Plenum Press, New York. 